\title{
Microfluidic ion stripper for removal of trifluoroacetic acid from mobile phases used in HILIC-MS of intact proteins
}

\author{
Sam Wouters $^{1} \cdot$ Sebastiaan Eeltink ${ }^{1} \cdot$ Rob Haselberg $^{2,3} \cdot$ Govert W. Somsen $^{2,3} \cdot$ Andrea F. G. Gargano $^{2,3}$
}

Received: 8 February 2021 / Revised: 2 May 2021 / Accepted: 18 May 2021 / Published online: 28 May 2021

(C) The Author(s) 2021

\begin{abstract}
Trifluoroacetic acid (TFA) is commonly used as mobile phase additive to improve retention and peak shape characteristics in hydrophilic interaction liquid chromatography (HILIC) of intact proteins. However, when using electrospray ionization-mass spectrometry (ESI-MS) detection, TFA may cause ionization suppression and adduct formation, leading to reduced analyte sensitivity. To address this, we describe a membrane-based microfluidic chip with multiple parallel channels for the selective post-column removal of TFA anions from HILIC. An anion-exchange membrane was used to physically separate the column effluent from a stripper flow solution comprising acetonitrile, formic acid, and propionic acid. The exchange of ions allowed the post-column removal of TFA used during HILIC separation of model proteins. The multichannel design of the device allows the use of flow rates of $0.2 \mathrm{~mL} / \mathrm{min}$ without the need for a flow splitter, using mobile phases containing $0.1 \%$ TFA (13 mM). Separation selectivity and efficiency were maintained (with minor band broadening effects) while increasing the signal intensity and peak areas by improving ionization and reducing TFA adduct formation.
\end{abstract}

Keywords Microfluidic chips $\cdot$ LC-MS $\cdot$ MS ion suppression $\cdot$ LC ion-pairing $\cdot$ TFA $\cdot$ Intact protein analysis

\section{Introduction}

A clear advantage of workflows that study intact proteins by LC-MS compared to bottom-up proteomics is their capacity of maintaining information about the distribution of proteoforms [1]. This includes, for example, sequence variants and posttranslational modifications (PTMs), such as glycosylation and phosphorylation [2]. Proteoforms of, e.g., industrial enzymes or therapeutic proteins may have a different activity. Therefore, increasing attention is currently devoted to developing LC-MS methods that allow characterization of proteins

Sebastiaan Eeltink

seeltink@vub.be

$\triangle$ Andrea F. G. Gargano

a.gargano@uva.nl

1 Department of Chemical Engineering, Vrije Universiteit Brussel (VUB), 1050 Brussels, Belgium

2 Center for Analytical Sciences Amsterdam, Science Park 904, 1098 XH Amsterdam, The Netherlands

3 Division of BioAnalytical Chemistry, Amsterdam Institute of Molecular and Life Sciences, Vrije Universiteit Amsterdam, de Boelelaan 1085, 1081 HV Amsterdam, The Netherlands using so-called top-down proteomics analytical workflows, i.e., leaving the protein intact during analysis [3].

Reversed-phase liquid chromatography (RPLC) using acidic water-acetonitrile ( $\mathrm{ACN}$ ) gradients is commonly used for the denaturing LC-MS analysis of intact proteins. In addition, hydrophilic interaction liquid chromatography (HILIC)MS of intact proteins has been gaining attention due to its unique selectivity, being complementary to RPLC and thereby providing possibilities for protein glycoform analysis. [4-8] Both in RPLC and HILIC, the use of ion-pairing agents is essential in order to circumvent adverse ionic interactions of proteins with the stationary phase and allow separations to be based solely only on hydrophobic (RPLC) or hydrophilic interactions (HILIC). Strong ion-pairing agents like trifluoroacetic acid (TFA) can modulate retention, improve separation efficiency, and minimize peak tailing. In HILIC, they also facilitate protein solubilization and recovery $[9,10]$.

However, TFA often impairs ESI-MS detection to an extent that may vary between mass spectrometry type and generation of instruments. Typically, TFA in the mobile phase causes a shift in the protein ion charge state distribution to lower charge states as compared to more volatile acids like formic acid. Moreover, the protein signal may be distributed over multiple TFA adducts [11]. This is exemplified in Fig. S1 
of the Supplementary Information (ESM). To address this, several post-column strategies to perform efficient LC-MS using TFA-containing mobile phases have been proposed. These approaches include dilution [12-14], electrophoretic mobility control [15], membrane-based dialysis [16, 17], and exposure of the nebulized LC effluent to gas vapors (e.g., ACN, propionic acid) during ESI $[18,19]$. Most of these reports are limited to low-flow applications, use flow splitters, or require complicated setups, hindering their general use. Protein-TFA adduct formation during ESI can be counteracted by applying (in-source) collision-induced dissociation at increased energies (e.g., $50 \mathrm{eV}$ or more) [20]. However, this approach does not counteract ion suppression and may lead to in-source fragmentation of the protein.

Previously, we have developed a single-channel microfluidic membrane suppressor for use in capillary ion chromatography [21, 22]. In this study, we describe the design and application of a multichannel microfluidic suppressor ( $\mu$ EMPIS: microfluidic ESI-enabling Mobile Phase IonStrip) for the selective removal of TFA ions from LC mobile phases, and exchange with propionic acid and formic acid. The performance of the $\mu$ EMPIS is evaluated for HILIC separations of selected intact proteins, investigating operating conditions and their influence on the dispersion and MS gains using separation flow rates up to $0.2 \mathrm{~mL} / \mathrm{min}$.

\section{Experimental}

\section{Chemicals}

Deionized water $(18.2 \mathrm{~m} \Omega)$ was obtained from a Milli-Q purification system (Millipore, Bedford, USA). Acetonitrile (ACN, MS grade), isopropanol (IPA, LC-MS grade), formic acid (FA), propionic acid (PA), and trifluoroacetic acid (TFA, MS grade) were obtained from Biosolve (Valkenswaard, The Netherlands). Cytochrome $C$ (Cyt $C$, equine heart, $\geq 95 \%$ ), carbonic anhydrase (CA, bovine erythrocytes, $\geq 95 \%$ ), lysozyme (Lys, chicken egg white, $95 \%$ ), myoglobin (Myo, horse heart, $>90 \%$ ), ribonuclease A (RnA, bovine pancreas Type I-A, $\geq 60 \%$ ), ribonuclease $\mathrm{B}$ ( $\mathrm{RnB}$, bovine pancreas, $\geq 80 \%$ ), transferrin (Trans, human, $\geq 98 \%$ ), and ubiquitin (Ubi, bovine erythrocytes, $\geq 98 \%$ ) were acquired from Sigma (Zwijndrecht, The Netherlands). Protein standard solutions $(1 \mathrm{mg} / \mathrm{mL})$ were prepared in deionized water.

\section{Fabrication of microfluidic TFA ion stripper ( $\mu$ EMPIS)}

The device was designed using AutoCAD (Autodesk, San Rafael, USA), machining jobs were programmed using PrimCAM (Primus Data, Einsiedeln, Switzerland), and parts were manufactured using an $\mathrm{M} 7$ Compact $\mathrm{CNC}$ micro mill (Datron, Mühltal, Germany). An aminated vinylbenzyl chloride anion-exchange membrane (Thermo Fisher Scientific, Sunnyvale, USA) was pre-hydrated in the stripping solution and placed between two cyclic olefin copolymer (COC) substrate plates (TOPAS grade 8007, Kunststofzentrale Leipzig, Leipzig, Germany) with dimensions $90 \times 20 \times 1.5 \mathrm{~mm}$, featuring a microfluidic network with bifurcating flow distributor, 8 parallel channels $(\mathrm{L}=60 \mathrm{~mm}$ ), and flow collector (all $200 \mu \mathrm{m}$ deep and $300 \mu \mathrm{m}$ wide). The device volume is $32 \mu \mathrm{L}$. The stack was placed in a custom-made PEEK holder with aluminum backing featuring NanoPort connections (Upchurch Scientific, Oak Harbor, USA). The holder was bolted together with controlled torque. Dimensions of the $\mu$ EMPIS device is $110 \times 40 \times 30 \mathrm{~mm}$. A schematic representation of the device and its operating principle is reported in Fig. 1.

\section{LC separations}

HILIC protein separations were performed on an Agilent HPLC 1290 Infinity II (Waldbronn, Germany), composed of a binary pump, autosampler, column thermostat, and variable wavelength detector, using an Agilent AdvanceBio Glycan Mapping column $(150 \times 2.1 \mathrm{~mm}, 1.8 \mu \mathrm{m}$ particle diameter with $300 \AA$ pore size) with a Phenomenex SecurityGuard Ultra Cartridge (Widepore C4; $2 \times 4.6 \mathrm{~mm}$ i.d.) (Utrecht, The Netherlands).

Gradient separations were performed using solvent A: 98\% ACN, $2 \%$ water, $0.1 \%$ TFA, and B: $88 \%$ water, $10 \%$ IPA, $2 \%$ ACN, $0.1 \%$ TFA. Gradient details are as follows: for intact proteins: a linear gradient from 20 to $50 \% \mathrm{~B}$ in $20 \mathrm{~min}$; for the separation of RnA and RnB: 20 to $30 \%$ B in 1 min followed by a linear gradient from 30 to $37 \% \mathrm{~B}$ in $20 \mathrm{~min}$. The flow rate and column temperature were $0.1 \mathrm{~mL} / \mathrm{min}$ and $60^{\circ} \mathrm{C}$, respectively, unless stated differently.

Different solvents and additives were used for the stripper flow and are described in the figures (Fig. 2 illustrates the effects of different stripper flow compositions; other experimental conditions tested are reported in section S3 of the ESM) of the respective experiments. The stripper flow rate was about 6 times the eluent flow rate.

\section{Mass spectrometry}

A Bruker Daltonics maXis HD high-resolution quadrupole time-of-flight (qTOF) mass spectrometer (Bremen, Germany) was used in positive-ion mode. Operating conditions are as follows: nebulizer: 0.8 bar; dry gas: $8 \mathrm{~L} / \mathrm{min}$; nitrogen dry temperature: $220{ }^{\circ} \mathrm{C}$; quadrupole-ion and collision cell energies: 5 and $10 \mathrm{eV}$, respectively; collision cell RF: $2000 \mathrm{Vpp}$; in-source CID (isCID): $30 \mathrm{eV}$; funnel RF: 400 Vpp; multipole RF: $800 \mathrm{Vpp}$; transfer and prepulse storage times: 190.0 and $20.0 \mu \mathrm{s}$, respectively. The monitored mass range was $300-4000 \mathrm{~m} / \mathrm{z}$. Data analysis was done using Compass data analysis (4.3) from Bruker and deconvolution 


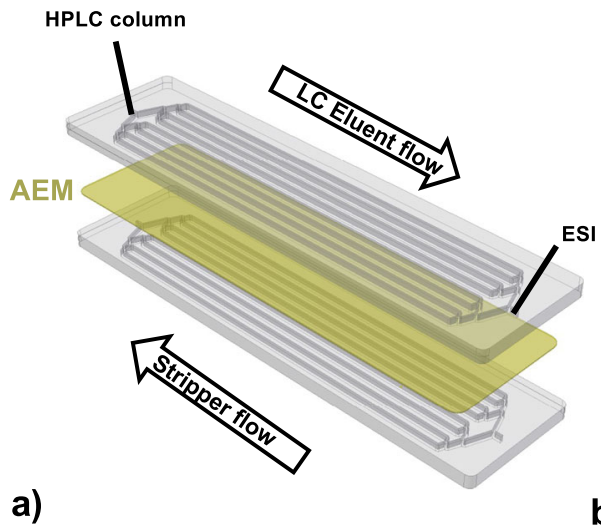

b)

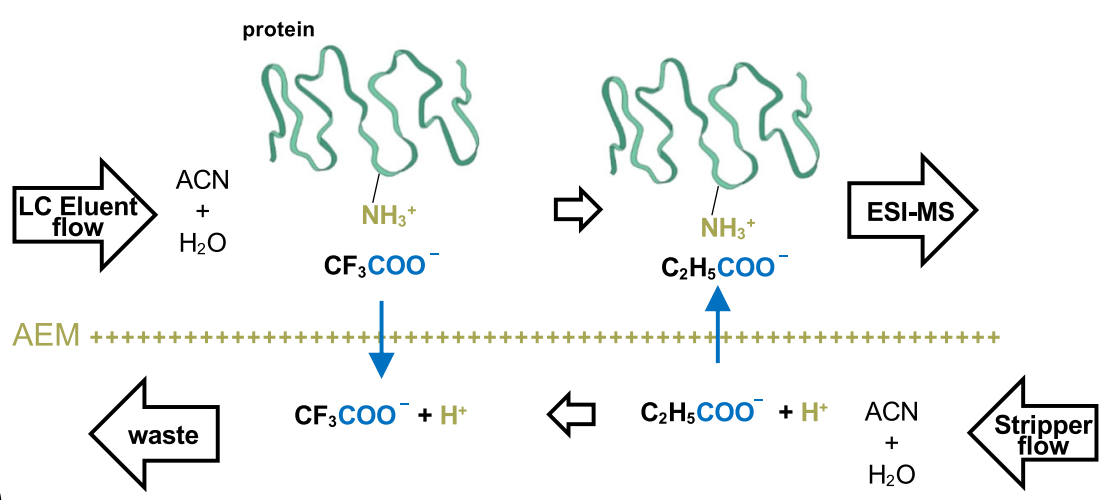

Fig. 1 a Schematic representation of the post-column microfluidic device for TFA removal ( $\mu$ EMPIS), showing the two COC chip substrates integrating eight open channels facing each other (gray) separated by an anion-exchange membrane (yellow). The eluent from the LC separation and the stripper flow run countercurrent in the two identical COC chip substrates. The channels are flushed from a single inlet to a single outlet using a flow distributor and collector having the same configuration. The outlet of the $\mu$ EMPIS is connected to ESI-MS. b Illustration of the ionexchange processes taking place in correspondence of the anionexchange membrane (AEM). The effluent flow after the HPLC separation contains TFA ions that are exchanged with PA ion carried in the stripper flow

time. Notably, the 8-channel design also provides essential support for the flexible membrane. When spanning a single wide channel of, e.g., $1 \mathrm{~mm}$ or more, such an unsupported flexible membrane could restrict the flow in the channels when pressure differences are introduced between the stripper flow side and the effluent side.

During its operation, TFA ions from the column effluent are exchanged for counterions, e.g., formate or propionate, provided by the stripper flow solution, which is applied in counterflow. TFA can diffuse through the membrane towards the stripper channel driven by the concentration gradient across the membrane, while the counterions migrate to the effluent channel to balance the charge (and to reduce its concentration gradient). The counter flow ensures that the concentration gradient of TFA between the channels is maximized near the outlet of the effluent channels and therefore favors the exchange of the residual TFA present in the effluent. The $\mathrm{pH}$ in the effluent remains low enough to keep the proteins positively charged, ensuring they are repulsed from the positively charged membrane surface. The presence of acetonitrile in the effluent minimizes hydrophobic interactions of the proteins with the membrane.

To asses potential protein losses due to adsorption on the membrane of the $\mu$ EMPIS device, we performed flow injection analysis using mobile phase composition that mimic the chromatographic conditions commonly used in denaturing chromatography. In particular, we used mobile phases having low (10\% ACN + 0.1\% TFA) and high percentages of ACN $(45 \% \mathrm{ACN}+0.1 \% \mathrm{TFA})$. Experiments were performed with and without device using reference proteins (BSA, trypsinogen and lysozyme) and UV detection (Table S1). The presence of high percentage of ACN (above 45\%) was important to ensure sufficient recovery. Recoveries were between 90 and $100 \%$ when using $45 \%$ of ACN (Table S1). Therefore, we 

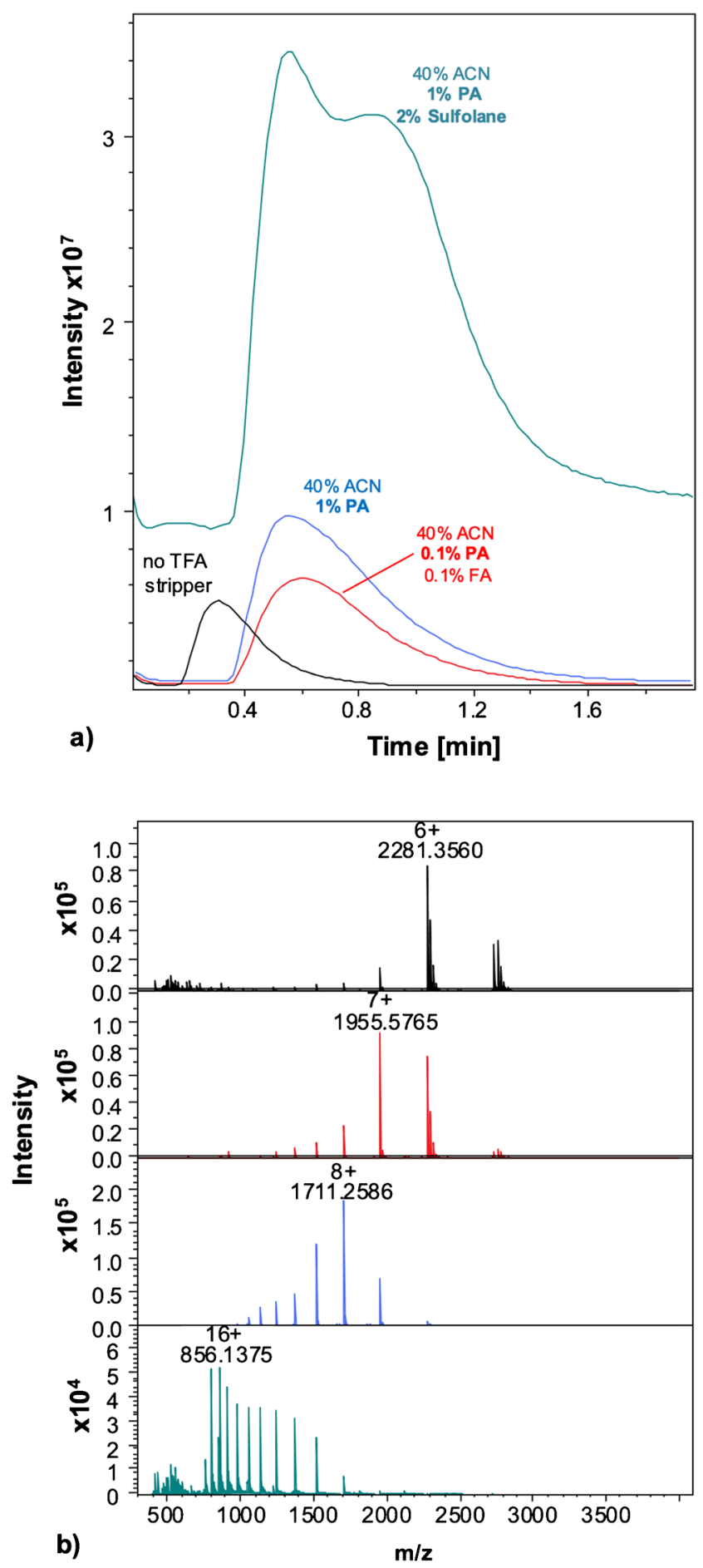

Fig. 2 Flow injection ESI-MS of RnA (0.1 mg/mL) in ACN-water 40:60 (v/v) containing $0.1 \%$ TFA. a TIC obtained for RnA without $\mu$ EMPIS (black) and with $\mu$ EMPIS using a stripper solvent of ACN-water 40:60 (v/v) containing $0.1 \%$ FA and $0.1 \%$ PA (red), $1 \%$ PA (blue), and $1 \%$ PA and $2 \%$ sulfolane (green). b Mass spectra obtained for RnA using the stripper conditions mentioned under a (corresponding colors). Other conditions: injection volume, $2 \mu \mathrm{L}$; eluent and stripper flow rate, $0.2 \mathrm{~mL} / \mathrm{min}$. Further conditions, see Experimental section. Extracted ion currents (EIC) for different RnA charge states under the different conditions in Fig. 3a are reported in Fig. S1 of the ESM. See Fig. S2 of the ESM for a closer comparison of the MS spectra with $1 \% \mathrm{PA}$ and $1 \%$ PA and $2 \%$ sulfolane decided to limit the scope of the device to HILIC applications where the ACN \% is maintained above $45 \%$.

After having established conditions that prevent protein adsorption, we used flow injection ESI-MS experiments to evaluate the effect of the experimental conditions (stripper flow composition, flow rate) on the efficiency of the $\mu$ EMPIS in increasing MS signal intensity and reducing adduct formation. This was done by injecting $2 \mathrm{uL}$ of solution of $\mathrm{RnA}$ in ACN-water 40-60 (v/v) containing 0.1\% TFA in an eluent of the same composition with a flow of $0.2 \mathrm{~mL} / \mathrm{min}$ and different stripper flow compositions. The stripper solutions were based on $40 \%$ ACN with various additives: $0.1 \%$ propionic acid (PA) and $0.1 \%$ formic acid (FA), $1 \%$ PA, and $1 \%$ PA and 2\% sulfolane. Both PA and sulfolane have been described as capable of reducing TFA signal suppression and adduct formation (propionic acid [7, 13, 19] and sulfolane [24]). The corresponding protein total ion chromatograms and mass spectra are reported in Fig. 2.

Under all conditions, the incorporation of the microfluidic stripper device increased the band width by about $1.27 \times$ (peak width at half height, FWHM, increased from 0.259 to $0.329 \mathrm{~min}$ ) as can be visually observed comparing the black trace with the other traces in Fig. 2a. The increase of peak width results from the increased dispersion volume introduced by the $\mu$ EMPIS device $(32 \mu \mathrm{L})$. However, the increased peak width was accompanied by an improvement on the MS detection in terms of signal increase and removal of TFA adducts. No increase of peak asymmetry (e.g., tailing) was observed (in both cases, we observed a value of approximately 1.7).

When using a stripper solvent containing $1 \%$ propionic acid (PA), a $2 \times$ increase of the TIC signal intensity for RnA was obtained (Fig. 2a). Moreover, the corresponding mass spectrum of RnA showed a shift to higher charge states (maximum of the charge state distribution shifting from $\left[\mathrm{M}+6 \mathrm{H}^{+}\right]^{6+}$ to $+[\mathrm{M}+$ $\left.\left.8 \mathrm{H}^{+}\right]^{8+}\right)$ as well as the absence of protein-TFA adduct peaks. Results obtained with a lower concentration of PA $(0.1 \%)$ showed a similar shift in protein charge state but had a reduced effect in terms of adduct removal. This suggests that a higher concentration gradient of PA is needed to favor the TFA exchange to compensate for the different $\mathrm{pKa}$ between the acids ( 0.23 for TFA vs $4.88 \mathrm{PA}$ ). Moreover, the introduction of PA in the ESI solvent is likely to reduce the ionization suppression and favor the removal of adducts during the ESI of the protein. The higher boiling point of PA with respect to TFA has been indicated as a possible reason for its favorable TFA adduct removal behavior $[12,18]$.

Additionally, we evaluated the use of a supercharging agent (sulfolane [25]) in the stripper flow to enhance the protein signal. Supercharging agents have shown potential in increasing MS signal when using TFA-based mobile phases. However, the direct addition of reagent like sulfolane directly in the mobile phases can create permanent changes to the column surface disrupting chromatographic performance [24]. 

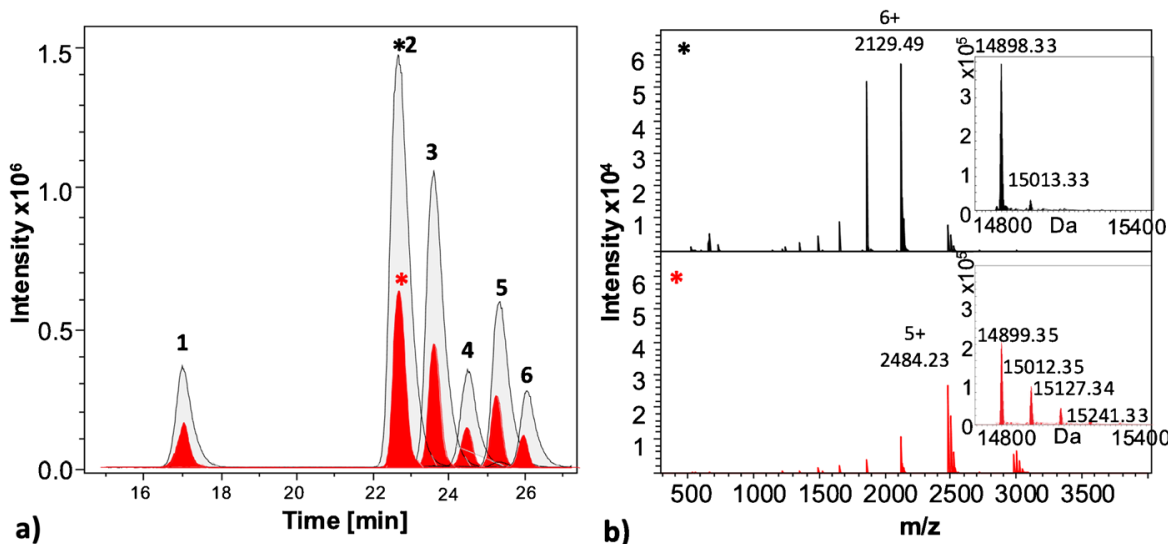

b)

Fig. 3 a Extracted ion current (EIC) of the ribonuclease glycoforms: RnA (1), RnB1 (2), RnB2 (3), RnB3 (4), RnB4 (5), RnB5 (6) obtained from a HILIC-MS run using of $0.4 \mu \mathrm{g}$ of a mixture of RnA and B with (black) and without $\mu$ EMPIS device (red). b Mass spectra of ribonuclease B1 (*) and inserts showing the corresponding deconvoluted mass spectra and the relative abundance of TFA adducts. The stripper flow composition was of $70 \%$ ACN with $0.1 \%$ FA and $1 \%$ PA. The runs with and without $\mu E M P I S$ are time-aligned to facilitate the comparison (shifted about $0.5 \mathrm{~min}$ ). A summary from the data from the extracted ion current for the single protein glycoforms is reported in Fig. S2 of the ESM. The EICs are obtained summing the intensities of the $m / z$ for 7+, 6+, 5+, 4+ mass of each proteoform of RnA. RnA $(1521.3,1711.2 ; 1955.6 ; 2281.3 \mathrm{~m} / \mathrm{z})$, RnB1 (1656.5; 1863.4; 2129.5; $2484.2 \mathrm{~m} / \mathrm{z})$, RnB2 (1674.5; 1883.7; $2152.6 ; 2511.2 \mathrm{~m} / \mathrm{z})$, RnB3 (1692.5; 1903.9; 2175.8; $2538.2 \mathrm{~m} / \mathrm{z})$, RnB4 (1710.5; 1924.2; 2198.9; $2565.3 \mathrm{~m} / \mathrm{z}$ ), RnB5 (1728.5; 1944.4; $2222.1 ; 2592.3 \mathrm{~m} / \mathrm{z}$ ). All EICs are extracted with a mass range of \pm $0.5 \mathrm{~m} / \mathrm{z}$. Other experimental conditions are reported in the experimental

A solution containing $2 \%(\mathrm{w} / \mathrm{v} ; \mathrm{g} / 100 \mathrm{~mL})$ sulfolane together with $1 \%$ propionic acid (PA) was mixed with the stripper flow, leading to a substantial increase $(15 \times)$ of the intensity of the TIC signal for RnA. A significant shift of the protein charge state distribution (maximum signal at +16 when using sulfolane as compared to +8 for the stripper flow solution containing $1 \%$ PA only) was observed (Figs. S2 and S3 of the ESM). However, the gain in MS signal when using sulfolane was compromised by an increase of the background signal. This is likely to be a consequence of the formation of protein-sulfolane adducts in the gas phase [26].

Interestingly, the TIC peak profile of the RnA peak presented a bimodal distribution. To better understand this last effect, we extracted ion currents from different charge states $(+17,+14$, and +10$)$. The corresponding extracted ion currents showed a bimodal distribution profile only for the higher charge states (results reported in ESM Fig. S4). This seems to exclude unspecific adsorption of the protein to the suppressor membrane due to the sulfolane (this should happen for all the protein charge states in case of adsorption) but to be a consequence of a change in the supercharging efficiency depending on the resulting from a different ratio between protein and supercharging agent. We concluded that sulfolane can be used in combination with the $\mu$ EMPIS device; however, including section. c EIC of the HILIC-MS separation of a protein mixture composed of (1) Ubi, (2) Cyt C, (3) Lys, (4) RnA, (5) RnB1, (6) RnB2 (each $0.2 \mathrm{mg} / \mathrm{mL} ; 1 \mu \mathrm{L}$ injected) with (black trace) and without (red trace) using the device. The TIC for the separation is reported in ESM Fig. S2; the chromatographic conditions are described in the Experimental section. The EICs are obtained summing the intensities of the $m / z$, for the main form of the protein. Ubi $(714.71 ; 779.50 ; 857.45 ; 952.50 ; 1071.56$; $1224.35 ; 1428.41 ; 1713.90 ; 2142.12 \mathrm{~m} / \mathrm{z})$, Cyt C (727.95; 773.39; $824.88 ; 883.72 ; 951.63 ; 1030.93 ; 1124.47 ; 1236.81 ; 1374.13 ; 1545.77$; $1766.44 ; 2060.68)$, Lys $(1192.96 ; 1301.32 ; 1431.45 ; 1590.39 ; 1788.94$; 2044.37; 2385.09), RnA (1521.33; 1711.25; 1955.57; 2281.33), RnB1 (1656.49; 1863.42; 2129.48; 2484.23), RnB2 (1674.49; 1883.67; 2152.63 ; 2511.24). All EICs are extracted with a mass range of \pm $0.5 \mathrm{~m} / \mathrm{z}$. Other experimental conditions are reported in the Experimental section. Results of the area, height, signal to noise, full width at half height are reported in Table 1. The corresponding TIC are in Fig. S5 of the ESM

this generated additional protein-sulfolane adducts and we preferred continuing our experiments excluding this.

Next, the effect of the flow rates of the eluent and stripper solvent was evaluated. Lowering eluent flow rates from 0.2 to $0.1 \mathrm{~mL} / \mathrm{min}$ increased the resident time of the analytes in the microfluidic device (by factor 2), allowing for a higher exchange rate and a corresponding signal increase (Fig. S4 of the ESM). Similarly, a higher stripper flow was beneficial (data not shown), helping to increase the concentration gradient between the two solvents. For the stripper solvent, a flow rate of about $6 \times$ the mobile phase flow rate was selected.

\section{Application of the $\mu$ EMPIS to HILIC-MS intact protein separations}

The conditions obtained from this optimization were used to apply the $\mu$ EMPIS device to the separation of protein mixtures. Here, we tested HILIC separations based on water-ACN mobile phases containing $0.1 \%$ TFA. Figure $3 a$ and $b$ show the HILIC-MS analysis of RnA and RnB employing a TFAcontaining eluent without (red trace) and with (black trace) the $\mu$ EMPIS device. The separation was run at $0.1 \mathrm{~mL} / \mathrm{min}$ using a gradient and a stripper flow of $0.6 \mathrm{~mL} / \mathrm{min}$ with a composition of $70 \% \mathrm{ACN}$ containing $0.1 \% \mathrm{FA}$ and $1 \% \mathrm{PA}$ (i.e., close to the HILIC separation conditions during elution). 
Table 1 Analysis of the increase of area, height, and full width at half height (FWHW) of the EIC the HILIC-MS analysis of proteins reported in Fig. 3. The results indicate the ratio of increase and are obtained by dividing the result (e.g., area) obtained with the $\mu$ EMPIS device with the one obtained without the device

Height (with $\mu$ EMPIS/

without $\mu$ EMPIS)

FWHM' (with $\mu$ EMPIS/ without $\mu$ EMPIS)

\begin{tabular}{lrrr}
\hline (1) Ubi & 2.68 & 0.91 & 2.96 \\
(2) Cyt C & 2.18 & 0.98 & 2.23 \\
(3) Lys & 32.24 & 13.25 & 2.24 \\
(4) RnA & 4.07 & 2.72 & 1.41 \\
(5) RnB1 & 5.23 & 3.31 & 1.47 \\
(6) RnB2 & 5.34 & 3.36 & 1.51 \\
\hline
\end{tabular}

In the HILIC separation, the different proteoforms of ribonuclease are resolved. The first eluting peak corresponds to the non-glycosylated protein (RnA) followed by its glycoforms $(\mathrm{RnB})$. RnB is N-glycosylated with a high mannose glycan consisting of 5-9 mannose units (RnB1-5, respectively). In HILIC, the different glycoforms are separated according to the number of mannose units (low to high). Notably, the addition of TFA is essential for achieving the efficient separation of the glycoforms of RnB; i.e., no glycoform resolution is obtained when FA is used instead in the mobile phase [5].

The extracted ion currents (EICs) in Fig. 2a are obtained summing the intensity of the protein peak in 4 charge states from +4 to $+7( \pm 0.5 \mathrm{~m} / \mathrm{z})$, allowing to remove the signals from the protein-TFA adducts. Our results show a clear improvement in sensitivity when using the $\mu$ EMPIS, with a peak area increase of about threefold and more than twofold in signal-tonoise ratio $(\mathrm{S} / \mathrm{N})$ (results reported in Table $\mathrm{S} 2$ of the ESM). Moreover, the obtained mass showed a shift of the protein ions to higher charge states when incorporating the TFA stripper (Fig. 3b). The TFA adducts account for about $50 \%$ of the deconvoluted protein signal (red spectra, insert in Fig. 3b) in the analysis without $\mu$ EMPIS, whereas they account for less than $10 \%$ when the device is present (black spectra, insert in Fig. 3b). Notably, the introduction of the $\mu$ EMPIS increases the peak widths (about $1.5 \times$, e.g., from 0.280 to $0.415 \mathrm{~min}$ FWHM for RnA peak), following the trend observed in FIA.

The peak area and width increased when using the $\mu$ EMPIS (Table 1; area from 3 and up to $32 \times$ and peak width between 1.5 and $3 \times$ ), while the asymmetry of the peaks did not change. The signal gain (area and height) varied depending on the protein. Proteins like Ubi and Cyt $\mathrm{C}$ did not present significant TFA adducts without the TFA stripper; therefore, the gain in signal was limited. Instead for RnA and Lys 4 to 8, TFA adducts were observed (Figs. S6 and S7 of the ESM). Here, the TFA stripper helped in reducing the dispersion of the signal, significantly increasing the height and area of the extracted ion current of the protein (i.e., $32 \times$ area and $13 \times$ height increase for Lys). Moreover, proteins eluting closer to the start of the gradient (at higher \% of $\mathrm{ACN}$ ) were more severely affected by the band broadening from the microfluidic device. This seems to indicate the presence of unspecific interactions with the $\mu$ EMPIS device that are somehow more relevant for less polar proteins (Ubi, Cyt C, eluting earlier in HILIC).

\section{Conclusions}

Here, we present proof of principle results demonstrating the feasibility of alleviating ion suppression by TFA-containing mobile phases in HILIC using a post-column multichannel microfluidic device ( $\mu$ EMPIS). The $\mu$ EMPIS allowed to exchange TFA ions across a anion-exchange membrane, replacing them with formic and propionic acid ions and favoring ionization. The use of the $\mu$ EMPIS device reduced the amount of protein-TFA adducts, but it did not eliminate the adducts for all the charge states. However, our results prove that signal improvement in terms of peak area and height can be achieved using this approach.

The capacity is one of the current limiting factors of the device. Fluid-dynamic investigations should be performed to maximize the exchange surface while minimizing dispersion, e.g., by making use of dual membranes, or hollow fibers. Moreover, membranes based on chemistries with reduced lipophilicity should be studied to reduce potential interactions with the membrane that lead to unwanted increase of peak widths and extend the application to RPLC-MS.

Supplementary Information The online version contains supplementary material available at https://doi.org/10.1007/s00216-021-03414-4.

Acknowledgements Prof. G. Desmet (Vrije Universiteit Brussel) is acknowledged for providing access to micro milling equipment.

Funding S. Wouters acknowledges financial support from the Flemish Agency for Innovation and Entrepreneurship. S. Eeltink acknowledges a research grant of the Research Foundation Flanders (FWO - grant no. 
G033018N). AG acknowledges financial support by the Netherlands Organization for Scientific Research, NWO Veni grant IPA (722.015.009)

\section{Declarations}

Conflict of interest The authors declare no competing interests.

Open Access This article is licensed under a Creative Commons Attribution 4.0 International License, which permits use, sharing, adaptation, distribution and reproduction in any medium or format, as long as you give appropriate credit to the original author(s) and the source, provide a link to the Creative Commons licence, and indicate if changes were made. The images or other third party material in this article are included in the article's Creative Commons licence, unless indicated otherwise in a credit line to the material. If material is not included in the article's Creative Commons licence and your intended use is not permitted by statutory regulation or exceeds the permitted use, you will need to obtain permission directly from the copyright holder. To view a copy of this licence, visit http://creativecommons.org/licenses/by/4.0/.

\section{References}

1. Smith LM, Kelleher NL. Proteoform: a single term describing protein complexity. Nat Methods. 2013;10(3):186-7 Available from: http://www.pubmedcentral.nih.gov/articlerender.fcgi?artid= 4114032\&tool=pmcentrez\&rendertype $=$ abstract.

2. Toby TK, Fornelli L, Kelleher NL. Progress in top-down proteomics and the analysis of proteoforms. Annu Rev Anal Chem. 2016;9(1):499-519 Available from: http://www.annualreviews. org/doi/10.1146/annurev-anchem-071015-041550.

3. Tran JC, Zamdborg L, Ahlf DR, Lee JE, Catherman AD, Durbin $\mathrm{KR}$, et al. Mapping intact protein isoforms in discovery mode using top-down proteomics. Nature. 2011;480(7376):254-8. https://doi. org/10.1038/nature10575.

4. Atri VD, Fekete S, Beck A, Lauber M, Guillarme D, D'Atri V, et al. Hydrophilic interaction chromatography hyphenated with mass spectrometry: a powerful analytical tool for the comparison of originator and biosimilar therapeutic monoclonal antibodies at the middle-up level of analysis. Anal Chem. 2017 [cited 2017 Feb 23];89(3):2086-92. Available from: http://pubs.acs.org/doi/ abs/10.1021/acs.analchem.6b04726.

5. Periat A, Fekete S, Cusumano A, Veuthey J-LL, Beck A, Lauber $\mathrm{M}$, et al. Potential of hydrophilic interaction chromatography for the analytical characterization of protein biopharmaceuticals. J Chromatogr A. 2016 [cited 2017 Feb 23];1448:81-92. Available from: http://www.sciencedirect.com/science/article/pii/ S0021967316304964\%5Cn. http://linkinghub.elsevier.com/ retrieve/pii/S0021967316304964.

6. Pedrali A, Tengattini S, Marrubini G, Bavaro T, Hemström P, Massolini G, et al. Characterization of intact neo-glycoproteins by hydrophilic interaction liquid chromatography. Molecules. 2014;19(7):9070-88.

7. Gargano AFG, Roca LS, Fellers RT, Bocxe M, Domínguez-Vega E, Somsen GW. Capillary HILIC-MS: a new tool for sensitive topdown proteomics. Anal Chem. 2018;90(11):6601-9. https://doi. org/10.1021/acs.analchem.8b00382.

8. Gargano AFG, Schouten O, van Schaick G, Roca LS, van den Berg-Verleg JH, Haselberg R, et al. Profiling of a high mannosetype $\mathrm{N}$-glycosylated lipase using hydrophilic interaction chromatography-mass spectrometry. Anal Chim Acta. 2020;1109: 69-77. https://doi.org/10.1016/j.aca.2020.02.042.

9. Garcia MC, Hogenboom ACC, Zappey H, Irth H, García MC, Hogenboom ACC, et al. Effect of the mobile phase composition on the separation and detection of intact proteins by reversed-phase liquid chromatography-electrospray mass spectrometry. J Chromatogr A. 2002;957(2):187-99. Available from: http://www. sciencedirect.com/science/article/pii/S002196730200345X.

10. Bobály B, Mikola V, Sipkó E, Márta Z, Fekete J. Recovery of proteins affected by mobile phase trifluoroacetic acid concentration in reversed-phase chromatography. J Chromatogr Sci. 2015;53(7): 1078-83.

11. Bobály B, Tóth E, Drahos L, Zsila F, Visy J, Fekete J, et al. Influence of acid-induced conformational variability on protein separation in reversed phase high performance liquid chromatography. J Chromatogr A. 2014;1325:155-62.

12. Apffel A, Fischer S, Goldberg G, Goodley PC, Kuhlmann FE. Enhanced sensitivity for peptide mapping with electrospray liquid chromatography-mass spectrometry in the presence of signal suppression due to trifluoroacetic acid-containing mobile phases. $\mathrm{J}$ Chromatogr A. 1995;712(1):177-90.

13. Kuhlmann FE, Apffel A, Fischer SM, Goldberg G, Goodley PC. Signal enhancement for gradient reversed-phase high-performance liquid chromatography-electrospray ionization mass spectrometry analysis with trifluoroacetic and other strong acid modifiers by postcolumn addition of propionic acid and isopropanol. J Am Soc Mass Spectrom. 1995;6:1221-5.

14. Chan CC, Bolgar MS, Dalpathado D, Lloyd DK. Mitigation of signal suppression caused by the use of trifluoroacetic acid in liquid chromatography mobile phases during liquid chromatography/ mass spectrometry analysis via post-column addition of ammonium hydroxide. Rapid Commun Mass Spectrom. 2012;26(12):1507-14.

15. Wang N-H, Lee W-L, Her G-R. Signal enhancement for peptide analysis in liquid chromatography-electrospray ionization mass spectrometry with trifluoroacetic acid containing mobile phase by postcolumn electrophoretic mobility control. Anal Chem. 2011;83(16):6163-8 Available from: http://pubs.acs.org/doi/abs/ $10.1021 /$ ac2003714.

16. New A P, Wolff JC, Crabtree S, Freitas do Santos L, Okafo G, Lee $\mathrm{J}$, et al. Preliminary investigation of the application of on-line membrane extraction of trifluoroacetic acid as an aid to improvement of negative ion electrospray mass spectrometry data. J Chromatogr A. 2001;913(1-2):205-8. Available from: http://www.ncbi.nlm.nih. gov/pubmed/11355814

17. Zhou Z, Zhang J, Xing J, Bai Y, Liao Y, Liu H. Membrane-based continuous remover of trifluoroacetic acid in mobile phase for LCESI-MS analysis of small molecules and proteins. J Am Soc Mass Spectrom. 2012;23(7):1289-92 Available from: https://link. springer.com/article/10.1007/s13361-012-0385-z.

18. Chen J, Liu Z, Wang F, Mao J, Zhou Y, Liu J, et al. Enhancing the performance of LC-MS for intact protein analysis by counteracting the signal suppression effects of trifluoroacetic acid during electrospray. Chem Commun. 2015;51(79):14758-60 Available from: http://www.ncbi.nlm.nih.gov/pubmed/26295950.

19. Wang S, Xing T, Li N, Yan Y, He Z, Liu AP, et al. Simple approach for improved LC-MS analysis of protein biopharmaceuticals via modification of desolvation gas. Anal Chem. 2019;91:3156-62.

20. Domínguez-Vega E, Tengattini S, Peintner C, van Angeren J, Temporini C, Haselberg R, et al. High-resolution glycoform profiling of intact therapeutic proteins by hydrophilic interaction chromatography-mass spectrometry. Talanta. 2018;184(January): 375-81. https://doi.org/10.1016/j.talanta.2018.03.015.

21. Wouters S, Bruggink C, Agroskin Y, Pohl C, Eeltink S. Microfluidic membrane suppressor module design and evaluation 
for capillary ion chromatography. J Chromatogr A. 2017;1484:2633. https://doi.org/10.1016/j.chroma.2016.12.078.

22. Wouters S, Wouters B, Jespers S, Desmet G, Eghbali H, Bruggink $\mathrm{C}$, et al. Design and performance evaluation of a microfluidic ionsuppression module for anion-exchange chromatography. J Chromatogr A. 2014;1355:253-60. https://doi.org/10.1016/j. chroma.2014.06.025.

23. Davydova E, Wouters S, Deridder S, Desmet G, Eeltink S, Schoenmakers PJ. Design and evaluation of microfluidic devices for two-dimensional spatial separations. J Chromatogr A. 2016 [cited 2017 Sep 7];1434:127-35. Available from: http:// linkinghub.elsevier.com/retrieve/pii/S0021967316000236.

24. Nshanian M, Lakshmanan R, Chen H, Loo RRO, Loo JA. Enhancing sensitivity of liquid chromatography-mass spectrometry of peptides and proteins using supercharging agents. Int J Mass Spectrom. 2018;427:157-64. https://doi.org/10.1016/j. ijms.2017.12.006.

25. Lomeli SH, Peng IX, Yin S, Ogorzalek Loo RR, Loo JA. New reagents for increasing ESI multiple charging of proteins and protein complexes. J Am Soc Mass Spectrom. 2010;21(1):127-31. https://doi.org/10.1016/j.jasms.2009.09.014.

26. Douglass KA, Venter AR. Investigating the role of adducts in protein supercharging with sulfolane. J Am Soc Mass Spectrom. 2012;23(3):489-97.

Publisher's note Springer Nature remains neutral with regard to jurisdictional claims in published maps and institutional affiliations. 